\title{
Expression Analysis of Four Peroxiredoxin Genes from Tamarix hispida in Response to Different Abiotic Stresses and Exogenous Abscisic Acid (ABA)
}

\section{Caiqiu Gao, Kaimin Zhang, Guiyan Yang and Yucheng Wang*}

State Key Laboratory of Forest Genetics and Tree Breeding, Northeast Forestry University, 26 Hexing Road, 150040 Harbin, China; E-Mail: chwcaogcq@yahoo.com.cn; zkm831@yahoo.cn (K.Z.); zhgxuygy@yahoo.com.cn (G.Y.)

* Author to whom correspondence should be addressed; E-Mail: ychngwang@yahoo.com; Tel.: +86-451-82190607-12; Fax: +86-451-82190607-11.

Received: 3 February 2012; in revised form: 3 March 2012 / Accepted: 6 March 2012 /

Published: 21 March 2012

\begin{abstract}
Peroxiredoxins (Prxs) are a recently discovered family of antioxidant enzymes that catalyze the reduction of peroxides and alkyl peroxides. In this study, four Prx genes (named as ThPrxII, ThPrxIIE, ThPrxIIF, and Th2CysPrx) were cloned from Tamarix hispida. Their expression profiles in response to stimulus of $\mathrm{NaCl}, \mathrm{NaHCO}_{3}, \mathrm{PEG}$, $\mathrm{CdCl}_{2}$ and abscisic acid (ABA) in roots, stems and leaves of T. hispida were investigated using real-time RT-PCR. The results showed that the four ThPrxs were all expressed in roots, stems and leaves. Furthermore, the transcript levels of ThPrxIIE and ThPrxII were the lowest and the highest, respectively, in all tissue types. All the ThPrx genes were induced by both $\mathrm{NaCl}$ and $\mathrm{NaHCO}_{3}$ and reached their highest expression levels at the onset of stress in roots. Under PEG and $\mathrm{CdCl}_{2}$ stress, the expression patterns of these ThPrxs showed temporal and spatial specificity. The expressions of the ThPrxs were all differentially regulated by ABA, indicating that they are all involved in the ABA signaling pathway. These findings reveal a complex regulation of Prxs that is dependent on the type of Prx, tissue, and the signaling molecule. The divergence of the stress-dependent transcriptional regulation of the ThPrx gene family in T. hispida may provide an essential basis for the elucidation of Prx function in future work.
\end{abstract}


Keywords: Prx gene; gene expression; Tamarix hispida; abiotic stresses; ABA

\section{Introduction}

Peroxiredoxins (Prxs), like catalase, superoxide dismutase, ascorbate peroxidase and glutathione peroxidase, are a group of prominent antioxidant enzymes in plants. They were first identified in 1996, when the Hv-1-CysPrx [1] and the Hv-2-CysPrx [2] were cloned from barley (Hordeum vulgare). Later, many Prxs were cloned and studied in other plants, such as Arabidopsis [3], rice (Oryza sativa) [4], liverwort (Riccia fluitans) [5], spinach (Spinacia oleracea) [6], poplar (Populus spp.) [7], tobacco (Nicotiana tabacum) [8] and winter rye (Secale cereale) [9]. Based on amino acid sequence similarities and specific structural features, mainly the number and position of conserved Cys residues, the Prx proteins have been grouped into four different families [10], namely 1-Cys Prx, 2-Cys Prx, type II Prx and type Q Prx. The members of 1-Cys Prx group have only one conserved Cys residue, and the 2-Cys Prx and type II Prx family members contain two conserved Cys residues, while the fourth group of Prx is the Q Prx type.

The function of Prxs in response to oxidative stresses has been studied in some depth. Prxs have also been reported to be involved in responses to other abiotic stresses, such as heat, cold, osmotic stress and high salinity [8,11-14]. Kim et al. [15] reported that the 2-Cys Prx (C2C-Prx1) from Chinese cabbage (Brassica rapa, subspecies pekinensis and chinensis) changed its protein structure from a low molecular weight (LMW) to a high molecular weight (HMW) complex against heat shock and oxidative stress. The 1-Cys Prx in Chinese cabbage also functions as a molecular chaperone under oxidative stress conditions [16]. Overexpression of At2-cys Prx in potato (Solanum tuberosum) enhanced tolerance to methyl viologen-mediated oxidative stress and high temperature [11]. Overexpression of PrxQ from Suaeda salsa $(S s P r x Q)$ in A. thaliana increased tolerance to salt and cold stress [17]. The transgenic maize overexpressing $\operatorname{Pr} x Q$ also showed the stress resistance against fungal disease and oxidative stress [8]. The transgenic tall fescue (Festuca arundinacea) overexpressing an Arabidopsis 2-Cys Prx is more tolerant against heat $\left(42{ }^{\circ} \mathrm{C}\right)$ and methyl viologen (MV) stress than the control plants, and with less electrolyte leakage and thiobarbituric acid-reactive substances (TBARS) [18].

Tamarix hispida is a shrub or small tree growing mainly in arid and semi-arid regions, which exhibits tolerance to salt, drought and high temperature. This makes T. hispida an ideal model plant for the investigation of physiological and molecular mechanisms of responses to stresses in trees and for the cloning of a stress tolerance gene. In the present study, four ThPrx genes, including three type II Prxs and one 2-Cys Prx gene, were cloned from T. hispida. To better understand the possible roles of ThPrx genes in abiotic stress tolerance, the expression profiles of these four ThPrx genes in response to the application of salt $(\mathrm{NaCl})$, salt- alkali $\left(\mathrm{NaHCO}_{3}\right)$, drought $(\mathrm{PEG})$, heavy metal $\left(\mathrm{CdCl}_{2}\right)$ and abscisic acid (ABA) in the root, stem and leaf tissue of T. hispida were monitored by real-time RT-PCR. 


\section{Results}

\subsection{Cloning and Sequence Analysis of Four ThPrx Genes}

Four ThPrx genes with complete open reading frames (ORFs) were identified from the six T. hispida libraries. The ORFs encoded deduced polypeptides of 162-274 amino acids, with a predicted molecular mass of 17.3-29.8 kDa and pI of 5.79-8.57 (Table 1). Except for ThPrxII, all ThPrx genes contain signal peptides with a length of 17-26 amino acids (Figure 1). Based on the number and position of conserved Cys residues, the Prxs were classified into four different types. According to this classification and the phylogenetic relationship among the Prx proteins, ThPrxII, ThPrxIIE and ThPrxIIF are type II Prx proteins, while Th2CysPrx belongs to 2-Cys Prx (Figure 2).

Table 1. Characteristics of the four ThPrxs from T. hispida.

\begin{tabular}{cccccc}
\hline Gene & $\begin{array}{c}\text { GenBank } \\
\text { accession } \\
\text { number }\end{array}$ & Type & $\begin{array}{c}\text { Deduced } \\
\text { number of } \\
\text { amino acid }\end{array}$ & $\begin{array}{c}\text { Isoelectric } \\
\text { point }\end{array}$ & $\begin{array}{c}\text { Molecular } \\
\text { mass (kDa) }\end{array}$ \\
\hline ThPrxII & JQ341201 & type II peroxiredoxin & 162 & 5.79 & 17.3 \\
Th2CysPrx & JQ341202 & 2Cys peroxiredoxin & 274 & 6.9 & 29.8 \\
ThPrxIIE & JQ341203 & type II peroxiredoxin & 227 & 8.57 & 24.0 \\
ThPrxIIF & JQ341204 & type II peroxiredoxin & 196 & 8.37 & 21.0 \\
\hline
\end{tabular}

Figure 1. Multiple sequence alignments of the four ThPrx proteins from T. hispida. Signal prediction analysis showed the three ThPrxs contain signal peptides with 17-26 amino acids. The signal peptide of each ThPrx is underlined.

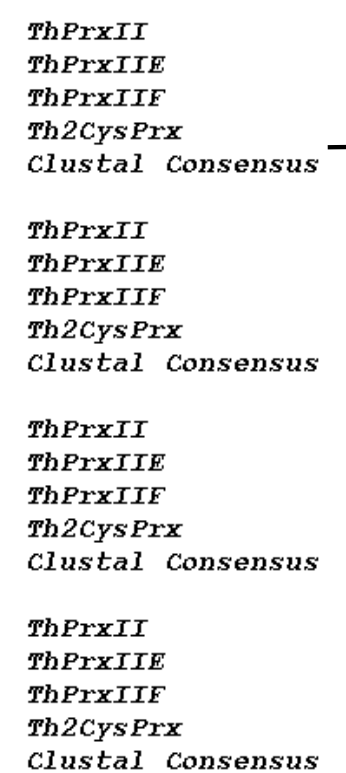

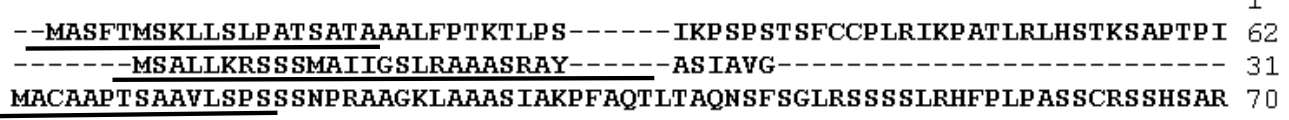


Figure 2. Phylogenetic tree of proteins homologous to Prx. Members of the four Prx proteins, including 2Cys-Prx, 1Cys-Prx, Prx-Q, and Type-II Prx, and in addition the Gpx family members were clustered with ClustalX. The first two letters correspond to the initials of the organism genus and species names. GenBank accession numbers are indicated: 1Cys-Prx: Arabidopsis, At1cysPrx (NP_175247); Brassica napus, Bn1cysPrx (AAF61460); Triticum turgidum, Tt1cysPrx (AAG50024). 2Cys-Prx: Arabidopsis, At2cysPrxA (NP_187769); Brassica napus, Bn2cysPrx (AAG30570); Nicotiana tabacum, Nt2cysPrx (CAC84143). Prx-Q: Arabidopsis, AtPrxQ (NP_189235); Gentiana triflora, GtPrxQ (BAD04985); Populus trichocarpa x Populus deltoides, PtPrxQ (AAS46230); Suaeda salsa, SsPrxQ (AAQ67661); Sedum lineare, SIPrxQ (BAA90524). Type-II Prx: Arabidopsis, AtPrxIIB (NP_176773); AtPrxIIC (NP_176772); AtPrxIID (NP_564763); AtPrxIIE (NP_190864); AtPrxIIF (NP_566268); Oryza sativa, OsPrxIIC (AAG40130); OsPrxIIE (BAA82377); Brassica rapa, BrPrxII (AF133302). GPX: Arabidopsis, AtGPX1 (NP_180080); AtGPX2 (NP_180715); AtGPX3 (NP_181863); AtGPX4 (NP_566128); Pisum sativum, PsGPX (sp|O24296); Lycopersicon esculentum, LeGPX (sp|O24031); Brassica napus, BnGPX (AAM12502).

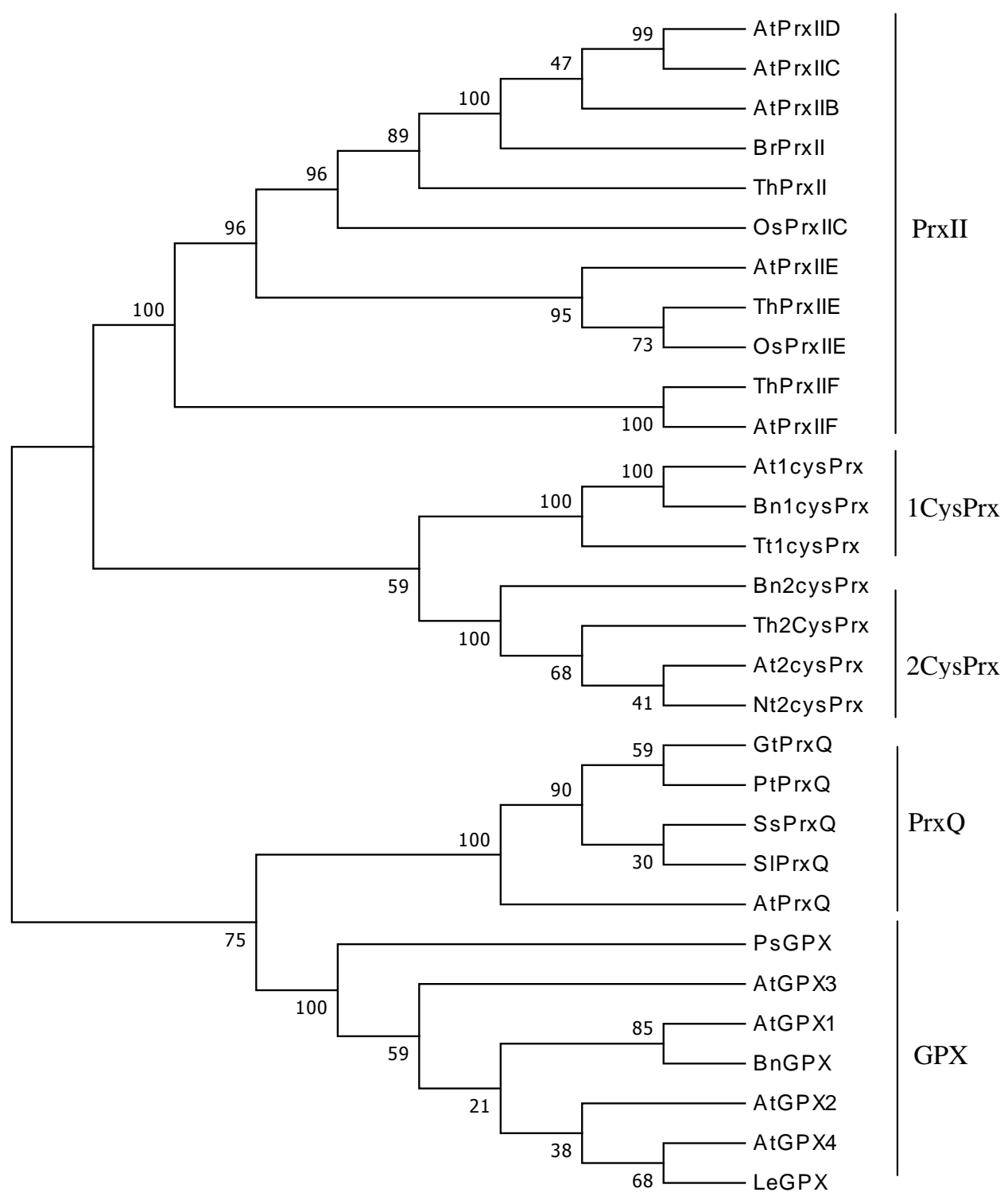


There were 14 ESTs representing the four unique ThPrx genes in the six libraries. The distribution of the 14 ThPrx ESTs was extremely heterogeneous in the libraries (Table 2). Among these ESTs, 12 ESTs were from the leaf libraries, and only 2 ESTs were identified in the root libraries. In addition, the number of ESTs representing different ThPrx genes in the library treated with $\mathrm{NaHCO}_{3}$ for $52 \mathrm{~h}$ increased one-fold compared with those in the library treated with $\mathrm{NaHCO}_{3}$ for $24 \mathrm{~h}$, indicating that the transcription of ThPrx genes may be up-regulated by $\mathrm{NaHCO}_{3}$ stress in leaves.

Table 2. The distribution of ThPrx ESTs among the T. hispida cDNA libraries.

\begin{tabular}{ccccccc}
\hline \multirow{2}{*}{ Gene } & \multicolumn{6}{c}{ Library } \\
\cline { 2 - 7 } & $0 \mathrm{~h}$ & $24 \mathrm{~h}$ & $48 \mathrm{~h}$ & $0 \mathrm{~h}$ & $24 \mathrm{~h}$ & $52 \mathrm{~h}$ \\
\cline { 2 - 7 } ThPrxII & 1 & 0 & 0 & 0 & 1 & 2 \\
Th2CysPrx & 0 & 0 & 0 & 0 & 0 & 1 \\
ThPrxIIE & 0 & 0 & 0 & 2 & 1 & 0 \\
ThPrxIIF & 0 & 0 & 1 & 1 & 1 & 3 \\
\hline Total & 1 & 0 & 1 & 3 & 3 & 6 \\
\hline
\end{tabular}

\subsection{Relative Expression Levels of Four ThPrx Genes in Roots, Stems and Leaves}

Relative expression levels of the four ThPrx genes in T. hispida roots, stems and leaves under normal growing condition were studied by using real-time PCR. The transcription level of the gene Actin was assigned as 100, and the transcription levels of ThPrx genes were plotted relative to the Actin gene transcription level (Table 3). The results indicated that these ThPrx were expressed in all tissues including roots, stems and leaves. Among these ThPrxs, the transcription levels of ThPrxIIE were the lowest in roots, stems and leaves, while the transcription levels of ThPrxII were the highest in all tissues. Transcription levels of Th2CysPrx were the second highest except in roots, where the relative abundance of Th2CysPrx and ThPrxIIF were nearly similar.

Table 3. Relative abundance of the four ThPrxs in different tissues of T. hispida. The transcription levels of the four ThPrx genes were plotted relative to the expression of Actin gene, and the transcription levels of Actin gene in root, stem and leaf were all assigned as 100.

\begin{tabular}{cccc}
\hline \multirow{2}{*}{ Gene } & \multicolumn{3}{c}{ Relative abundance } \\
\cline { 2 - 4 } & Roots & Stems & Leaves \\
\hline ThPrxII & 83.4 & 155.6 & 226.9 \\
Th2CysPrx & 17.8 & 42.3 & 187.9 \\
ThPrxIIE & 5.6 & 6.3 & 30.4 \\
ThPrxIIF & 20.1 & 15.0 & 38.4 \\
Actin & 100 & 100 & 100 \\
\hline
\end{tabular}

\subsection{Expression Profiles of ThPrx Genes in Response to Various Stresses}

In order to study the relationship between the ThPrxs genes and stress response of T. hispida, the expression patterns of the four ThPrx genes in response to different abiotic stresses $\left(\mathrm{NaCl}, \mathrm{NaHCO}_{3}\right.$, PEG and $\mathrm{CdCl}_{2}$ ) and ABA application were investigated using real-time PCR. 


\subsection{1. $\mathrm{NaCl}$ Stress}

In roots, all four ThPrx genes with the exception of ThPrxIIF were highly induced by $\mathrm{NaCl}$ stress at all treatment times, with the highest transcription levels being induced more than 56.8-fold (Figure 3A). The transcription levels of ThPrxII, ThPrxIIE and ThPrxIIF reached their peak levels at an early time point $(6 \mathrm{~h})$. Th2 CysPrx reached its highest transcription level at $24 \mathrm{~h}$, with the second highest levels at $6 \mathrm{~h}$. In stems, except for ThPrxII being down-regulated at $6 \mathrm{~h}$, the other three ThPrxs genes were all induced throughout the treatment period. However, the induction rate in stems was lower than that in roots. The most highly induced gene was ThPrxIIE, which was induced 4.96 -fold at $72 \mathrm{~h}$ of stress. In leaves, the expression patterns of the four ThPrxs genes were different. For instance, ThPrxII was down-regulated by $\mathrm{NaCl}$ stress. The transcription of ThPrxIIF was not altered at the early treatment stage and down-regulated at a later stage. Th2CysPrx was down-regulated at 6 and $24 \mathrm{~h}$ but was up-regulated at 12, 48 and $72 \mathrm{~h}$. ThPrxIIE was up-regulated at all time points.

Figure 3. Transcription analysis of the four ThPrxs responding to $\mathrm{NaCl}$ and $\mathrm{NaHCO}_{3}$ stress in roots, stems and leaves. The relative transcription level $=$ transcription level under stress treatment/transcription level under control condition $(0 \mathrm{~h})$. All relative transcription levels were $\log 2$-transformed. Error bars (SD) were obtained from nine replicates of the real-time PCR that included three biological replicates and each biological replicate contains three technical replicates. A: $\mathrm{NaCl}$ stress; $\mathbf{B}: \mathrm{NaHCO}_{3}$ stress.

A

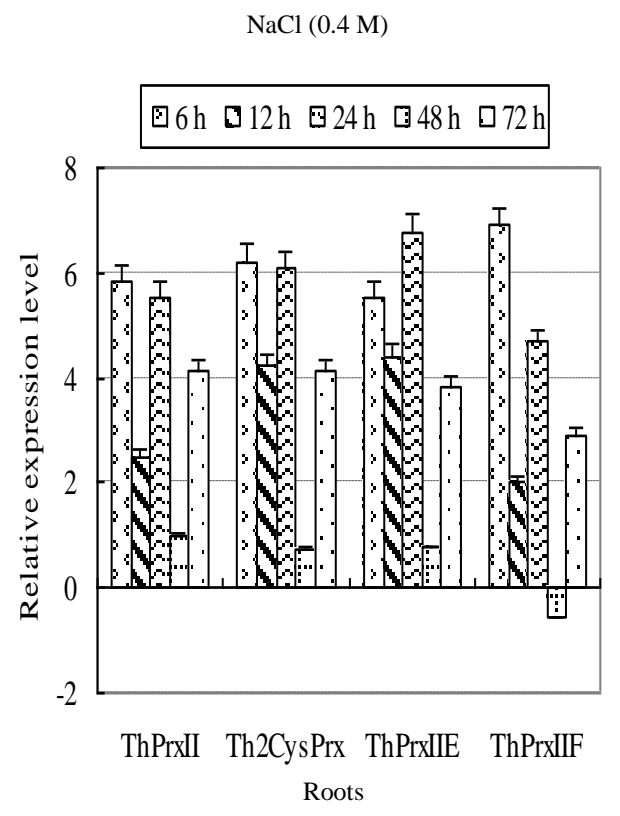

B

$\mathrm{NaHCO}_{3}(0.3 \mathrm{M})$

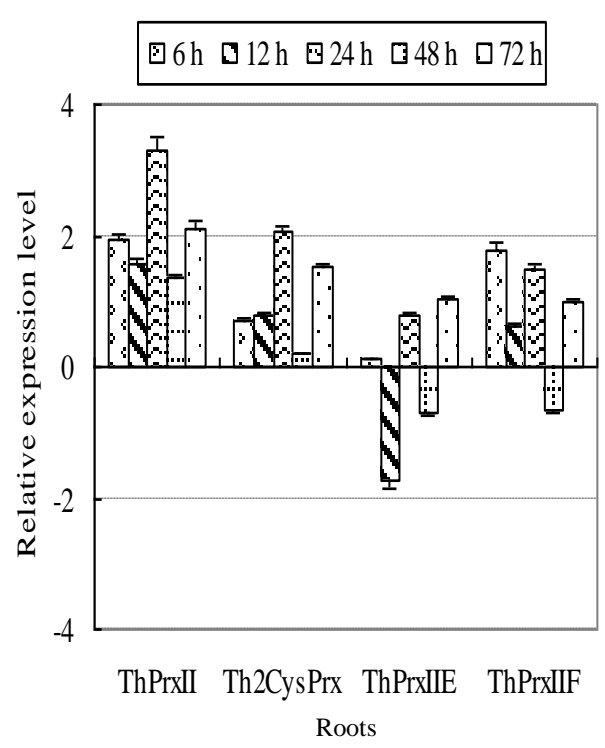


Figure 3. Cont.
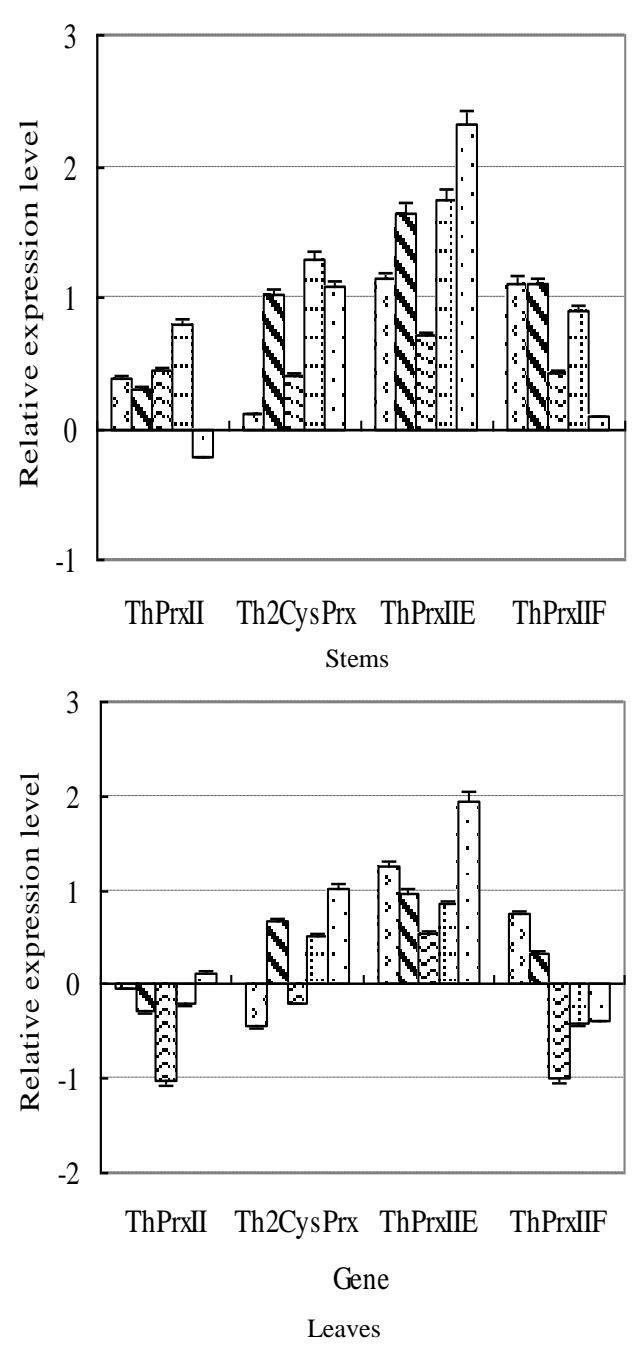
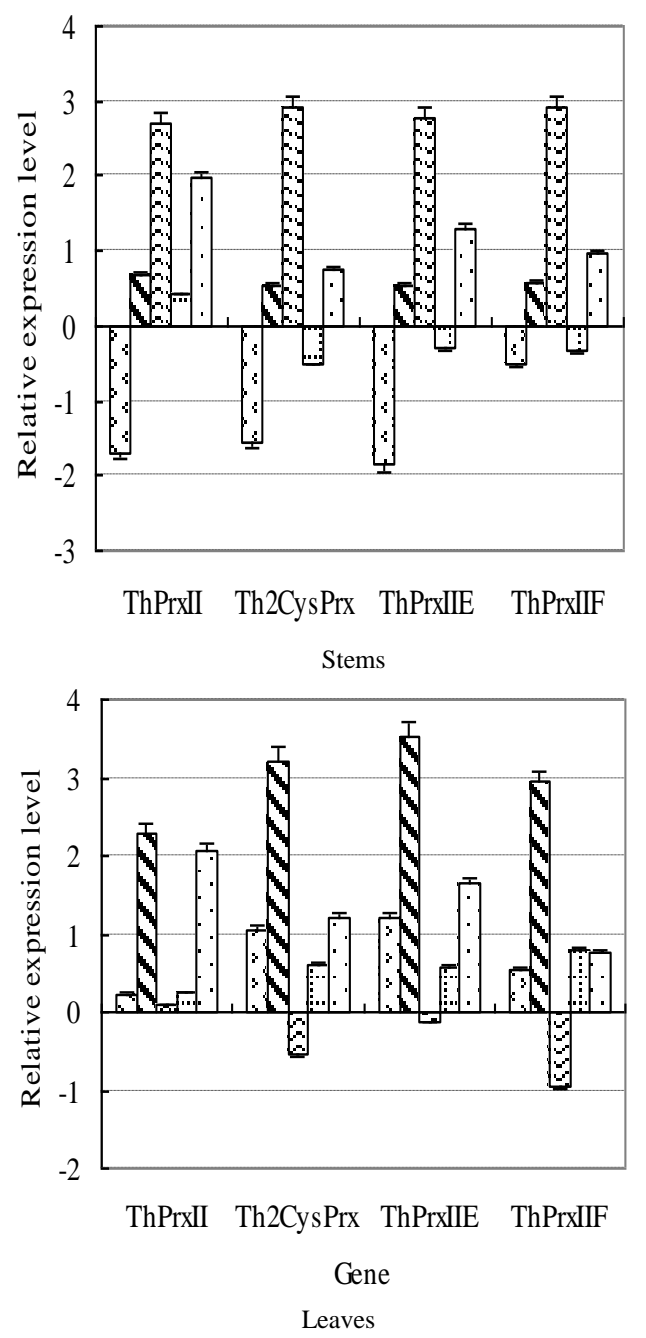

\subsection{2. $\mathrm{NaHCO}_{3}$ Stress}

In roots, all four ThPrx genes were up-regulated at most time points; especially ThPrxII and Th2CysPrx which were induced throughout the entire treatment period. In stems, all genes were down-regulated at an early time point $(6 \mathrm{~h})$, followed by up regulation. They all reached their peak expression levels at $24 \mathrm{~h}$ of stress. In leaves, the four ThPrx genes were induced at an early time point, and reached their highest expression levels at $12 \mathrm{~h}$. All genes were down-regulated at $24 \mathrm{~h}$. Furthermore, the expression levels were equal to or lower than their levels at $0 \mathrm{~h}$ (Figure 3B).

\subsubsection{PEG Stress}

In roots, the transcription levels of ThPrxII, ThPrxIIE and ThPrxIIF were generally decreased, while Th2CysPrx at most time points was up-regulated. In stems, ThPrxII was down-regulated, while the other three ThPrxs were all up-regulated. In leaves, all four ThPrx gene transcriptions were downregulated (Figure 4A). 


\subsection{4. $\mathrm{CdCl}_{2}$ Stress}

The transcription levels of the four ThPrx genes were divided into two distinct groups. One group contained ThPrxIIE and ThPrxIIF, and they shared similar expression patterns, while ThPrxII and Th2CysPrx constituted the other group. In roots, ThPrxIIE and ThPrxIIF, were up-regulated during the $\mathrm{CdCl}_{2}$ stress period, except at $72 \mathrm{~h}$. In contrast, ThPrxII and Th2CysPrx were down-regulated for at least three time points. The transcription level of $\operatorname{ThPrx} 1$ decreased by $58 \%$ at $72 \mathrm{~h}$, when compared with levels at $0 \mathrm{~h}$. In stems, ThPrxIIE and ThPrxIIF were up-regulated at all stress times. ThPrxII and Th2CysPrx levels showed alternating up- and down-regulation patterns. In leaves, the expression pattern was opposite to stems. ThPrxII and Th2CysPrx were down-regulated, while ThPrxIIE and ThPrxIIF levels showed alternating up- and down-regulation patterns (Figure 4B).

Figure 4. Expression analysis of the four ThPrxs responding to application of abscisic acid (ABA), and treatments of PEG and $\mathrm{CdCl}_{2}$ in roots, stems and leaves. The relative transcription level $=$ transcription level under stress treatment/transcription level under control condition $(0 \mathrm{~h})$. All relative transcription levels were $\log 2$-transformed. Error bars (SD) were obtained from nine replicates of the real-time PCR that included three biological replicates and each biological replicate contains three technical replicates. A: PEG stress; B: $\mathrm{CdCl}_{2}$ stress; $\mathbf{C}$ : ABA treatment.

A

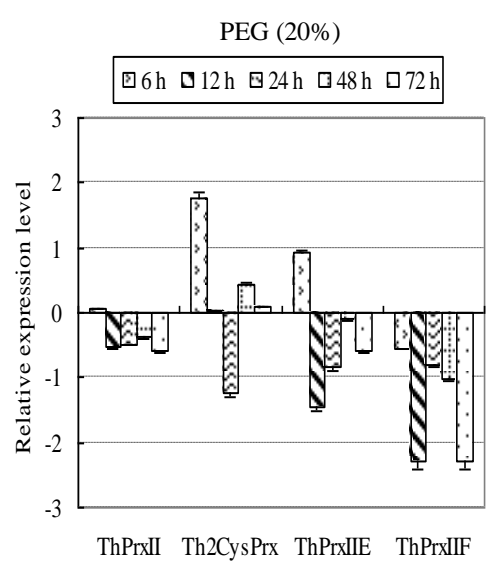

Roots

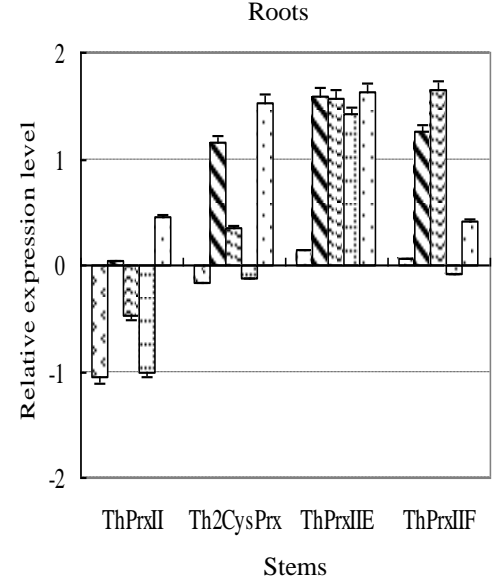

$\mathrm{B}$
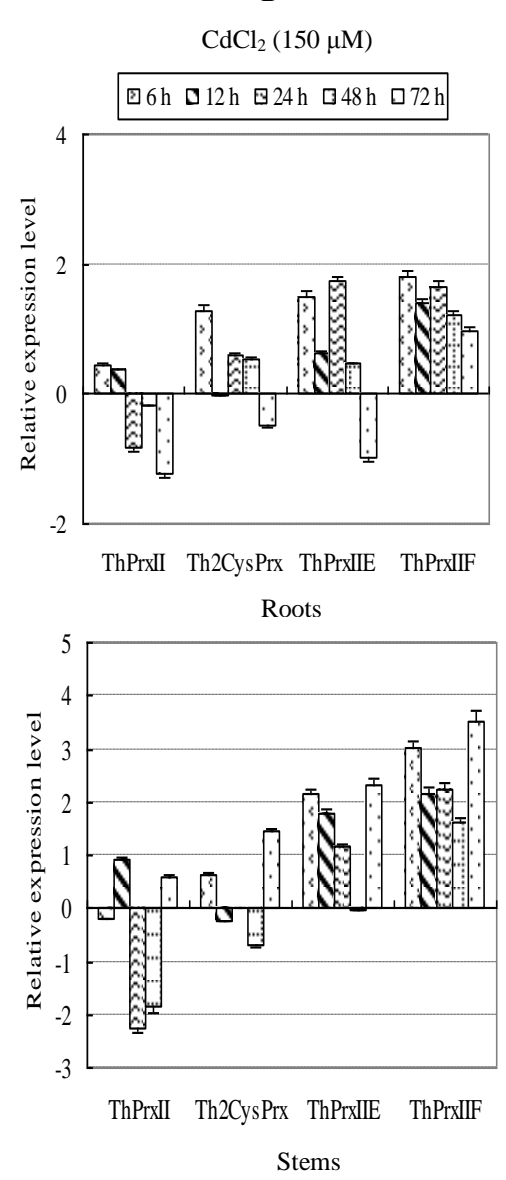

C

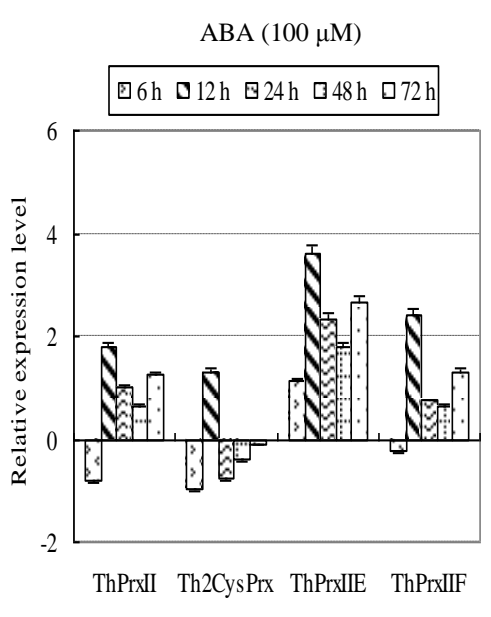

Ronte

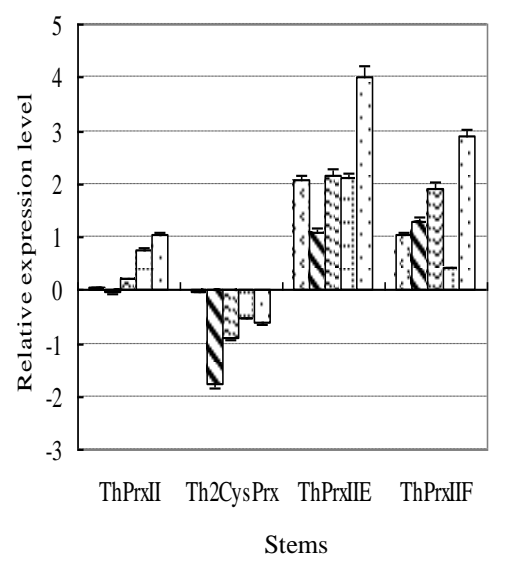


Figure 4. Cont.
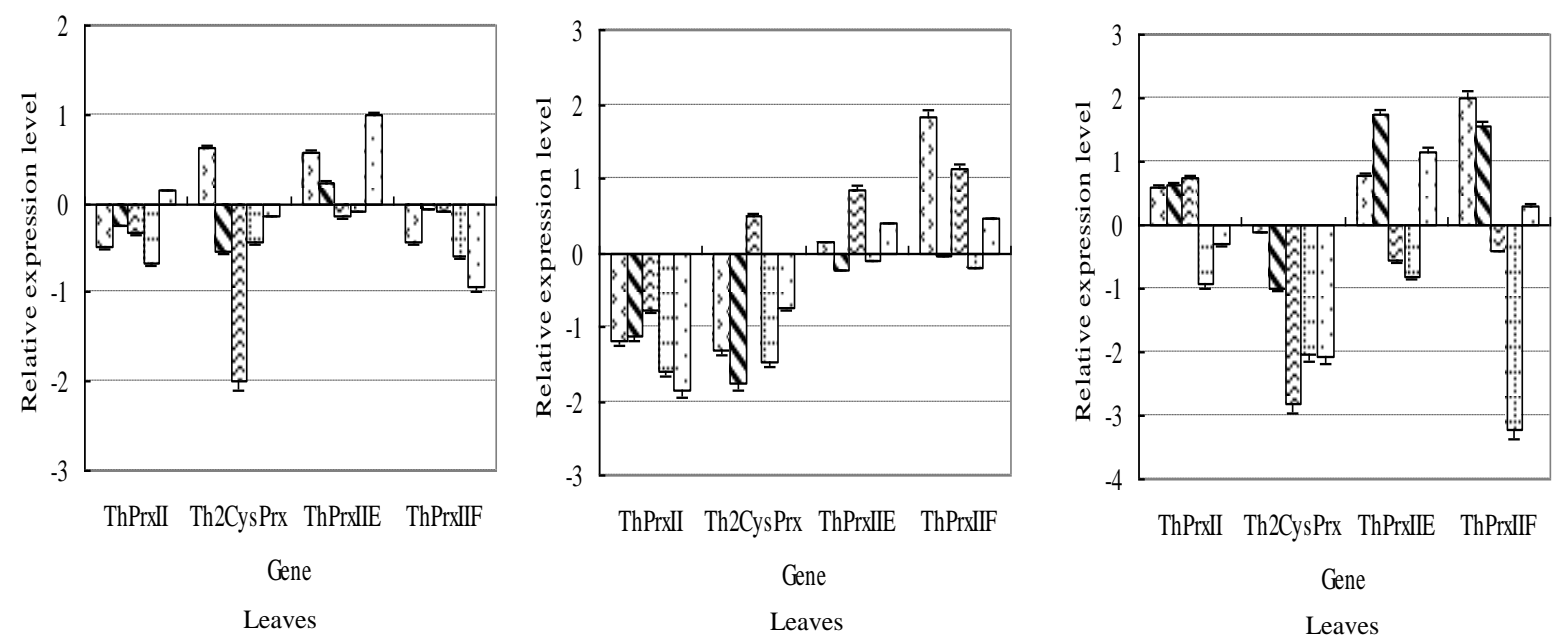

\subsubsection{ABA Application}

The RT-PCR results demonstrated that the four ThPrx genes shared similar expression patterns in roots, stems and leaves under the regulation of ABA. ThPrxII, ThPrxIIE and ThPrxIIF were up-regulated at most time points. In contrast, Th2CysPrx transcription was down-regulated, especially in stems and leaves (Figure 4C).

\section{Discussion}

The Prx gene family is ubiquitously distributed in all organisms from bacteria to higher plants. It is a small gene family with only 10 genes in Arabidopsis and 11 genes in rice. In this study, we cloned four Prx genes with complete ORFs from T. hispida, including three type II Prxs and one 2-Cys Prx. The transcription levels of the four ThPrx genes were notably different under normal growth conditions (Table 4). Th2CysPrx, as the other type Prx gene, was also abundant in stems and leaves. Especially in leaves, where its abundance was slightly lower than that of ThPrxII. Muthuramalingam et al. [19] confirmed that the 2-Cys peroxiredoxin can act as a regulatory hub in the chloroplast; therefore, Th2CysPrx may also act as a key peroxiredoxin in chloroplast.

Here, we have shown that these four ThPrx genes are induced by at least two types of abiotic stresses, indicating that all four ThPrx genes may play roles in abiotic stress response of T. hispida. Previous studies demonstrated that the transcription of Prx genes are in response to different kinds of stresses, such as low or high light, salinity, heavy metals, nutrient deprivation, temperature extremes and chemical effectors [20]. For example, the transcript levels of the 1-Cys Prx gene from Xerophyta viscosa Baker increased when subjected to dehydration, heat $\left(42{ }^{\circ} \mathrm{C}\right)$, high light intensity $\left(1500 \mu \mathrm{mol}\right.$ photons $\left.\mathrm{m}^{-2} \cdot \mathrm{s}^{-1}\right), \mathrm{NaCl}(100 \mathrm{mM})$ and $100 \mu \mathrm{M}$ ABA [21]. Finkemeier et al. [12] observed that the transcripts of AtPrxII $F$ were increased in roots of Arabidopsis under $\mathrm{CdCl}_{2}$ stress. AtPrxIIC transcript levels responded strongly during oxidative stress [22]. In pea leaves, PrxII $F$ protein accumulates upon cold and heavy-metal treatment [23].

Interestingly, under drought (20\% PEG) and heavy metal $\left(\mathrm{CdCl}_{2}\right)$ stress conditions, the four $\operatorname{ThPrx}$ genes displayed different expression patterns. Furthermore, they showed different responses to 
different stress types, times and organ-specific variations. At the same time, all four ThPrx genes were induced by both salt $(\mathrm{NaCl})$ and salt-alkali $\left(\mathrm{NaHCO}_{3}\right)$ stress. In roots, all four genes reached their highest transcription levels at an early $\mathrm{NaCl}$ stress stage $(6 \mathrm{~h})$, suggesting that the transcripts of these ThPrx genes are triggered rapidly under stress conditions. Prx proteins are found to play a central role in ROS detoxification [23]; therefore, these ThPrxs that are all highly induced by stresses may also play important roles in ROS detoxification in cells under salt stress.

Dietz et al. [20] summarized and demonstrated that $\operatorname{Pr} x$ transcript regulation varied, depending on the type of Prx, plant species, stress intensity, and developmental state. Consistent with these results, Th2CysPrx, the unique 2-Cys Prx gene among the studied four ThPrxs, showed the most peculiar expression pattern under different stress conditions and application of ABA. Its transcription was stimulated upon an increase in the roots after PEG stress and decreased in all organs by ABA application. The divergence of the stress-dependent expressional regulation among the four ThPrx genes in T. hispida suggest that, compared with the other ThPrx genes, Th2CysPrx may play different roles in resistance to stress or may be involved in different signaling transduction processes. Until now, expressional regulation of $\operatorname{Prx}$ on promoter and signaling levels has only been investigated for At1-CysPrx and At2-CysPrx [24-25]. Therefore it is essential to study the expressional regulation of ThPrxs in response to stress tolerances on the promoter and signaling levels in the future.

\section{Experimental Section}

\subsection{Plant Materials and Stress Treatments}

Seedlings of $T$. hispida were grown in pots containing a mixture of turf peat and sand $(2: 1 \mathrm{v} / \mathrm{v})$ in a greenhouse under controlled conditions of 70-75\% relative humidity, light/dark cycles of 14/10 h with lights on at 7.00 AM, and maintaining a temperature of $24^{\circ} \mathrm{C}$. Two month-old seedlings were used for experimental analysis. To induce abiotic stresses, the seedlings were watered into their roots with solutions of $0.4 \mathrm{M} \mathrm{NaCl}, 0.3 \mathrm{M} \mathrm{NaHCO}_{3}, 20 \%$ (w/v) PEG6000 and $150 \mu \mathrm{M} \mathrm{CdCl}_{2}$ for $0,6,12,24,48$ and $72 \mathrm{~h}$, respectively. For ABA treatment, the seedlings were exposed to $100 \mu \mathrm{M}$ ABA solution $(0.1 \% v / v$ ethanol). The ABA solution was watered into the roots of seedlings. For the control, the seedlings were exposed with the same volume of water containing only the same concentration of ethanol without ABA. The leaves, stems and roots from at least 24 seedlings were collected and pooled after various stress time points $(0,6,12,24,48$ and $72 \mathrm{~h})$, immediately frozen in liquid nitrogen and stored at $-80{ }^{\circ} \mathrm{C}$ until further use. Three samples from each treatment were prepared for real-time PCR biological repeats.

\subsection{Cloning and Identification of $4 \mathrm{ThPrx}$ Genes}

Six cDNA libraries were constructed including three samples from $T$. hispida leaves [26] and three from T. hispida roots [27]. In total, 17,173 ESTs were obtained from the six libraries. The ESTs were assembled into singletons and contigs, with the parameters set at 40 bp overlap and $95 \%$ identity using the CAP3 assembly program. The functional annotation of ESTs was performed using BLASTX and BLASTN against the non-redundant (NR) NCBI database. The ESTs representing the four ThPrx genes were identified according to their functional annotations. The library clones containing the Prxs 
genes were further sequenced from both sides to confirm their sequences and to generate complete sequence data.

\subsection{Sequence Alignments and Phylogenetic Analysis}

All four ThPrx protein sequences were aligned by ClustalX. The Prx proteins from T. hispida and other plants were subjected to phylogenetic analysis by conducting a phylogenetic tree reconstruction employing the neighbor-joining (NJ) method in ClustalX. Furthermore, the classification of the four ThPrx genes was carried out according to the classification and designation method of Bréhélin et al.[9]. Signal peptide predictions for the four ThPrx proteins were performed using the Signal peptide tool (http://www.cbs.dtu.dk/services/SignalP/). Molecular weight (MW) and isoelectric point (pI) predictions for every deduced ThPrxs were carried out by the Compute $\mathrm{pI} / \mathrm{Mw}$ tool (http://www.expasy.org/tools/protparam.html).

\subsection{RNA Extraction and Reverse Transcription (RT)}

Total RNA was isolated from leaves, roots or stems using a CTAB method and digested with DNase I (Promega, Madison, WI, USA) to remove any DNA residue. Approximately $0.5 \mu \mathrm{g}$ of total RNA was reversely transcribed (RT) into cDNA using an oligodeoxythymidine primer and six random primers in a final reaction volume of $10 \mu \mathrm{L}$ following the PrimeScript ${ }^{\mathrm{TM}}$ RT reagent Kit protocol (TaKaRa). The synthesized cDNAs were diluted to $100 \mu \mathrm{L}$ with sterile water and used as the template for real-time RT-PCR.

\subsection{Real-time Quantitative RT-PCR}

Real-time RT-PCR was performed using an MJ Opticon ${ }^{\mathrm{TM}^{2}}$ machine (Biorad, Hercules, CA, USA). The genes of alpha tubulin (FJ618518), beta tubulin (FJ618519) and beta actin (FJ618517) were chosen as internal controls (reference gene) to normalize the amount of total RNA present in each reaction. The $20 \mu \mathrm{L}$ reaction mixture contained $10 \mu \mathrm{L}$ of SYBR-Green Real-time PCR Master Mix (Toyobo), $0.5 \mu \mathrm{M}$ of specific primers for ThPrx genes (Table 4) or reference genes, and $2 \mu \mathrm{L}$ cDNA template (equivalent to $100 \mathrm{ng}$ of total RNA).

Table 4. Primer sequences used for quantitative RT-PCR analysis

\begin{tabular}{lll}
\hline Gene & \multicolumn{1}{c}{ Forward Primers (5'-3') } & \multicolumn{1}{c}{ Reverse Primers $\left(5^{\prime}-3^{\prime}\right)$} \\
\hline ThPrxII & TCAGCAGGTTCAGTTCACT & CAGAGCCATCAGCAAGGA \\
Th2CysPrx & GAGAAGGCTTGGACTGAG & GAGGAACGGCAAGATGAG \\
ThPrxIIE & CCCTCTCCTATTTGACTCC & TCAGCAGCAGGACTTCATC \\
ThPrxIIF & CTTCCTCATCGGAAATATGTCG & AACAAACACCTGTGTACGCACC \\
Actin & AAACAATGGCTGATGCTG & ACAATACCGTGCTCAATAGG \\
$\alpha$-tubulin & CACCCACCGTTGTTCCAG & ACCGTCGTCATCTTCACC \\
$\beta$-tubulin & GGAAGCCATAGAAAGACC & CAACAAATGTGGGATGCT \\
\hline
\end{tabular}

The amplification was completed with the following cycling parameters: $94{ }^{\circ} \mathrm{C}$ for $30 \mathrm{~s}$, followed by 45 cycles at $94{ }^{\circ} \mathrm{C}$ for $12 \mathrm{~s}, 60{ }^{\circ} \mathrm{C}$ for $30 \mathrm{~s}, 72{ }^{\circ} \mathrm{C}$ for $40 \mathrm{~s}$ and $1 \mathrm{~s}$ at $81{ }^{\circ} \mathrm{C}$ for plate reading. A 
melting curve was generated for each sample at the end of each run to assess the purity of the amplified products. For each sample, reactions were carried out in triplicate to ensure the reproducibility of the results. The gene transcription levels of the clones were calculated from the threshold cycle according to $2^{-\Delta \Delta \mathrm{Ct}}[28]$.

\section{Conclusions}

In conclusion, we cloned four ThPrx genes with complete ORFs from T. hispida, including three type II Prxs and one 2-Cys Prx. Expression analysis showed that these four ThPrx genes are all expressed in the roots, stem and leaves, suggesting that they play roles in all of these tissues. In addition, these four genes are all associated with abiotic stress responses and are involved in the ABA signaling pathway. Consequently, some of these ThPrx genes may have potential for use in the genetic improvement of abiotic stress tolerance in plants.

\section{Acknowledgments}

This work was supported by National Natural Science Foundation of China (No. 31000312), the Specialized Research Fund for the Doctoral Program of Higher Education of China (No. 20100062120001) and the Fundamental Research Funds for the Central Universities (No. DL12CA03).

\section{References}

1. Stacy, R.A.; Munthe, E.; Steinum, T.; Sharma, B.; Aalen, R.B. A peroxiredoxin antioxidant is encoded by a dormancy related gene, Per1, expressed during late development in the aleurone and embryo of barley grains. Plant Mol. Biol. 1996, 31, 1205-1216.

2. Baier, M.; Dietz, K.J. Primary structure and expression of plant homologues of animal and fungal thioredoxin-dependent peroxide reductases and bacterial alkyl hydroperoxide reductases. Plant Mol. Biol. 1996, 31, 553-564.

3. Pulido, P.; Spínola, M.C.; Kirchsteiger, K.; Guinea, M.; Pascual, M.B.; Sahrawy, M.; Sandalio, L.M.; Dietz, K.J.; González, M.; Cejudo, F.J. Functional analysis of the pathways for 2-Cys peroxiredoxin reduction in Arabidopsis thaliana chloroplasts. J. Exp. Bot. 2010, 61, 4043-4054.

4. Umate, P. Genome-wide analysis of thioredoxin fold superfamily peroxiredoxins in Arabidopsis and rice. Plant Signal Behav. 2010, 5, 1543-1546.

5. Horling, F.; Baier, M.; Dietz, K.J. Redox-regulation of the expression of the peroxide- detoxifying chloroplast 2-cys peroxiredoxin in the liverwort Riccia. fluitans. Planta 2001, 214, 304-313.

6. Baier, M.; Dietz, K.J. The plant 2-Cys peroxiredoxin BAS1 is a nuclear-encoded chloroplast protein: its expressional regulation, phylogenetic origin, and implications for its specific physiological function in plants. Plant J. 1997, 12, 179-190.

7. Rouhier, N.; Gelhaye, E.; Sautiere, P.E.; Brun, A.; Laurent, P.; Tagu, D.; Gerard, J.; de Fay, E.; Meyer, Y.; Jacquot, J.P. Isolation and characterization of a new peroxiredoxin from poplar sieve tubes that uses either glutaredoxin or thioredoxin as a proton donor. Plant Physiol. 2001, 127, 1299-1309. 
8. Kiba, A.; Nishihara, M.; Tsukatani, N.; Nakatsuka, T.; Kato, Y.; Yamamura, S. A peroxiredoxin $\mathrm{Q}$ homolog from gentians is involved in both resistance against fungal disease and oxidative stress. Plant Cell Physiol. 2005, 46, 1007-1015.

9. Berberich, T.; Uebler, M.; Feierabend, J. Cloning of a cDNA encoding a thioredoxin peroxidase (TPx) homolog from winter rye (Secale cereale L.) (Accession No. AF076920) (PGR98-167). Plant Physiol. 1998, 118, 711.

10. Bréhélin, C.; Meyer, E.H.; de Souris, J.P.; Bonnard, G.; Meyer, Y. Resemblance and dissemblance of Arabidopsis type II peroxiredoxins: similar sequences for divergent gene expression, protein localization, and activity. Plant Physiol. 2003, 132, 2045-2057.

11. Kim, M.D.; Kim, Y.H.; Kwon, S.Y.; Jang, B.Y.; Lee, S.Y.; Yun, D.J.; Cho, J.H.; Kwak, S.S.; Lee, H.S. Overexpression of 2-cysteine peroxiredoxin enhances tolerance to methyl viologen-mediated oxidative stress and high temperature in potato plants. Plant Physiol. Biochem. 2011, 49, 891-897.

12. Finkemeier, I.; Goodman, M.; Lamkemeyer, P.; Kandlbinder, A.; Sweetlove, L.J.; Dietz, K.J. The mitochondrial type II peroxiredoxin $\mathrm{F}$ is essential for redox homeostasis and root growth of Arabidopsis thaliana under stress. J. Biol. Chem. 2005, 280, 12168-12180.

13. Haslekås, C.; Viken, M.K.; Grini, P.E.; Nygaard, V.; Nordgard, S.H.; Meza, T.J.; Aalen, R.B. Seed 1-cysteine peroxiredoxin antioxidants are not involved in dormancy, but contribute to inhibition of germination during stress. Plant Physiol. 2003, 133, 1148-1157.

14. Lee, K.O.; Jang, H.H.; Jung, B.G.; Chi, Y.H.; Lee, J.Y.; Choi, Y.O.; Lee, J.R.; Lim, C.O.; Cho, M.J.; Lee, S.Y.; Rice 1Cys-peroxiredoxin over-expressed in transgenic tobacco does not maintain dormancy but enhances antioxidant activity. FEBS Lett. 2000, 486, 103-106.

15. Kim, S.Y.; Jang, H.H.; Lee J.R.; Sung, N.R.; Lee, H.B.; Lee, D.H.; Park, D.J.; Kang, C.H.; Chung, W.S.; Lim, C.O.; et al. Oligomerization and chaperone activity of a plant 2-Cys peroxiredoxin in response to oxidative stress. Plant Sci. 2009, 177, 227-232.

16. Kim, S.Y.; Paeng, S.K.; Nawkar, G.M.; Maibam, P.; Lee, E.S.; Kim, K.S.; Lee, D.H.; Park, D.J.; Kang, S.B.; Kim, M.R.; et al. The 1-Cys peroxiredoxin, a regulator of seed dormancy, functions as a molecular chaperone under oxidative stress conditions. Plant Sci. 2011, 181,119-124.

17. Jing, L.W.; Chen, S.H.; Guo, X.L.; Zhang, H.; Zhao, Y.X. Overexpression of a chloroplast-located peroxiredoxin Q gene, SsPrxQ, increases the salt and low-temperature tolerance of Arabidopsis. J. Integr. Plant Biol. 2006, 48, 1244-1249.

18. Kim, K.H.; Alam, I.; Lee, K.W.; Sharmin, S.A.; Kwak, S.S.; Lee, S.Y.; Lee, B.H. Enhanced tolerance of transgenic tall fescue plants overexpressing 2-Cys peroxiredoxin against methyl viologen and heat stresses. Biotechnol Lett. 2010, 32, 571-576.

19. Muthuramalingam, M.; Seidel, T.; Laxa, M.; Nunes de Miranda, S.M.; Gärtner, F.; Ströher, E.; Kandlbinder, A.; Dietz, K.J.Multiple redox and non-redox interactions define 2-Cys peroxiredoxin as a regulatory hub in the chloroplast. Mol. Plant. 2009, 2, 1273-1288.

20. Dietz, K.J. Peroxiredoxins in Plants and Cyanobacteria. Antioxid. Redox Signal. 2011, 15, 1129-1159.

21. Mowla, S.B.; Thomson, J.A.; Farrant, J.M.; Mundree, S.G. A novel stress-inducible antioxidant enzyme identified from the resurrection plant Xerophyta viscosa Baker. Planta 2002, 215, 716-726. 
22. Horling, F.; Lamkemeyer, P.; König, J.; Finkemeier, I.; Kandlbinder, A.; Baier, M.; Dietz, K.J. Divergent light-, ascorbate-, and oxidative stress-dependent regulation of expression of the peroxiredoxin gene family in Arabidopsis. Plant Physiol. 2003, 131, 317-325.

23. Barranco-Medina, S.; Krell, T.; Bernier-Villamor, L.; Sevilla, F.; Lazaro, J.J.; Dietz, K.J. Hexameric oligomerization of mitochondrial peroxiredoxin PrxIIF and formation of an ultrahigh affinity complex with its electron donor thioredoxin Trx-o. J. Exp. Bot. 2008, 59, 3259-3269.

24. Haslekås, C.; Grini, P.E.; Nordgard, S.H.; Thorstensen, T.; Viken, M.K.; Nygaard, V.; Aalen, R.B. ABI3 mediates expression of the peroxiredoxin antioxidant AtPER1 gene and induction by oxidative stress. Plant Mol. Biol. 2003, 53, 313-326.

25. Baier, M.; Ströher, E.; Dietz, K.J. The acceptor availability at photosystem I and ABA control nuclear expression of 2-Cys peroxiredoxin-A in Arabidopsis thaliana. Plant Cell Physiol. 2004, 45, 997-1006.

26. Gao,C.; Wang, Y.; Liu G.;, Yang, C.; Jiang, J.; Li, H. Expression profiling of salinity-alkali stress responses by large-scale expressed sequence tag analysis in Tamarix hispid. Plant Mol. Biol. 2008, $66,245-258$.

27. Li, H.; Wang, Y.; Jiang, J.; Liu, G.; Gao, C.; Yang, C. Identification of genes responsive to salt stress on Tamarix hispida roots. Gene 2009, 433, 65-71.

28. Livak, K.J.; Schmittgen, T.D. Analysis of relative gene expression data using real-time quantitative PCR and the 2(-Delta Delta C(T)) Method. Methods 2001, 25, 402-408.

(C) 2012 by the authors; licensee MDPI, Basel, Switzerland. This article is an open access article distributed under the terms and conditions of the Creative Commons Attribution license (http://creativecommons.org/licenses/by/3.0/). 